\title{
Objective Measures of Vest Therapy Adherence Among Pediatric Subjects With Cystic Fibrosis
}

\author{
Christine M Benoit, Eric Christensen, Amanda J Nickel, Sophie Shogren, Mahrya Johnson, \\ Elizabeth Franck Thompson, and John McNamara
}

\begin{abstract}
BACKGROUND: Airway clearance techniques (ACTs), including high-frequency chest wall oscillation (vest therapy), are important for maintaining lung function for patients with cystic fibrosis, but daily completion of ACTs is time-consuming and cumbersome. Patient adherence is a persistent challenge, and adherence data are largely patient reported, which may reduce accuracy. To provide definitive adherence data, this study utilized a Bluetooth-enabled vest therapy system to remotely collect objective adherence data from a cohort of pediatric subjects. METHODS: Vest usage data were collected over a 12-month period and compared to each subject vest prescription. Because vest prescriptions are multifaceted, we developed metrics to examine adherence to the various facets (eg, daily treatment, treatments per day, treatment duration, frequency settings, and frequency and pressure settings combined) as well as an overall adherence metric. RESULTS: Among the 73 enrolled subjects, 62 (50\% male, age range 2-19 y) completed the study. Among adolescent subjects age 13$19 \mathrm{y}$, average adherence to daily vest therapy $(\mathbf{4 4 . 0 \%})$ was significantly lower than that among subjects 0-6 y old $(77.8 \%)$ and subjects $7-12$ y old $(89.5 \%)$. As more prescribed therapy components were combined into a single adherence metric, all age groups declined in mean adherence rates, with overall adherence a decreasing function of age. CONCLUSIONS: Employing new technology to remotely collect vest usage data allows for a granular examination of vest therapy adherence. While maintaining high levels of treatment adherence becomes increasingly difficult as children age, we also found substantial reductions in adherence rates among all age groups when more complex aspects of therapy prescriptions, such as frequency and pressure settings, were examined. These data illustrate areas providers and care teams can focus on to improve patient adherence to vest prescriptions. Key words: cystic fibrosis; child; adolescent; adherence; high-frequency chest wall oscillation. [Respir Care 2020;65(12):1831-1837. (C) 2020 Daedalus Enterprises]
\end{abstract}

\section{Introduction}

With improvements in diagnostic techniques and more effective treatment options available, the life expectancy of patients with cystic fibrosis has improved markedly over

Ms Benoit, Ms Nickel, Ms Shogren, Ms Thompson, and Dr McNamara are affiliated with the Children's Minnesota Research Institute, Minneapolis, Minnesota. Dr Christensen is affiliated with the College of Continuing and Professional Studies, University of Minnesota, Minneapolis, Minnesota. Ms Johnson is affiliated with the Department of Psychiatry and Behavioral Sciences, University of Minnesota, Minneapolis, Minnesota. Dr McNamara is affiliated with the Children's Respiratory and Critical Care Specialists, Minneapolis, Minnesota.

Ms Benoit presented a version of this paper at the North American Cystic Fibrosis Conference, held November 2-4, 2017, in Indianapolis, Indiana. the past 20 years. ${ }^{1-4}$ Airway clearance techniques (ACTs) and devices, including high-frequency chest wall oscillation (ie, vest therapy), are widely recommended as the standard of care for cystic fibrosis to combat pulmonary disease caused by chronic airway inflammation and infection. ${ }^{5}$ Despite the central role ACTs play in improving pulmonary secretion clearance and helping to maintain lung

At the time of this study, Dr Christensen and Ms Johnson were affiliated with the Children's Minnesota Research Institute, Minneapolis, Minnesota.

This study was funded by an internal research grant from the Children's Minnesota Research Institute. All vest systems, technical support, and data server management were provided by Hill-Rom. The authors have disclosed no other conflicts of interest. 


\section{Vest Therapy Adherence Among Children With CF}

function, patient adherence is challenging because these therapies are time-consuming and cumbersome for patients to complete daily. Studies have reported low adherence to ACTs in all age groups (ie, children, ${ }^{6-8}$ adolescents,,${ }^{8,9}$ and adults ${ }^{10-12}$ ), with adherence declining as patients age and their daily routines become more complex.

A recent review of the literature revealed considerable discrepancies in self-reported ACT adherence rates compared to rates measured by objective tools. ${ }^{1}$ Despite ACT being widely recommended, evidence of its effectiveness is lacking, and there is disagreement among those who treat cystic fibrosis as to the benefits of vest therapy in particular. The absence of objective adherence measures has thus far limited the evidence base on ACT effectiveness. ${ }^{1,13}$ Previous attempts to obtain objective adherence data measurements from patients with cystic fibrosis are limited. In a recent study examining vest therapy adherence, Mikesell et $\mathrm{al}^{14}$ used a recording device integrated into a vest therapy system to monitor date of use, time of day, and duration of use. Their study showed that objective data can be collected; however, the recording devices used in their trial had a limited storage capacity and required home visits by a technician to download the data manually. Remote monitoring of vest therapy would eliminate these data collection challenges.

Our study used Bluetooth-enabled vest therapy to expand on the foundational work of Mikesell et $\mathrm{al}^{14}$ to remotely collect comprehensive vest usage data to determine subject adherence rates across a range of prescription components: daily treatment, treatments per day, duration of treatment, and vest settings. We hypothesized that adherence rates would decline with age and as more components of the vest therapy prescription were jointly considered.

\section{Methods}

\section{Subjects}

Subjects were recruited from the Children's Minnesota Cystic Fibrosis Clinic. Eligibility criteria included a documented history of cystic fibrosis, age $\geq 2 \mathrm{y}$, vest therapy prescription for a minimum of 12 months prior to enrollment, and current use of vest therapy. We categorized subjects into 3 age groups to reflect the level of presumed parental involvement with vest therapy: 2-6 y, with high parent involvement; 7-12 y, with moderate parent involvement; and 13-19 y, little to no parent involvement.

Supplementary material related to this paper is available at http://www. rcjournal.com.

Correspondence: Christine M Benoit PhD. Email: christine.benoit@ childrensmn.org.

DOI: $10.4187 /$ respcare. 07421

\section{QUICK LOOK}

\section{Current knowledge}

Patients with cystic fibrosis are often prescribed numerous daily medications and treatments to manage this lifelong, medically complex condition. High-frequency chest wall oscillation therapy is a common component of this daily regimen, despite being burdensome. Selfreported rates of adherence therapy vary widely, and objective measurements are limited.

\section{What this paper contributes to our knowledge}

We used a Bluetooth-enabled high-frequency chest wall oscillation device and transmitter to remotely collect objective therapy data automatically, including the number of treatments per day, duration of use, and frequency and pressure settings during use. Data were collected over a 12-month period and compared to prescription data to determine adherence rates among a cohort of pediatric subjects. Overall, adherence rates were lowest among teenagers, but all age groups had declining adherence rates as the more complex components of prescription data (ie, frequency and pressure settings) were taken into account.

The study was reviewed and approved by the institutional review board at Children's Minnesota. We obtained written consent from subjects $\geq 18$ y of age, written assent and parental consent for subjects 7-17 y old, and parental consent for those $<7 \mathrm{y}$ old.

\section{Data}

All subjects enrolled in the study received a Bluetoothenabled vest system (The Vest(c) Airway Clearance System, Model 105, Hill-Rom, Chicago, Illinois) and a wireless transmitter for remote transmission of vest use data. Vest usage data were automatically downloaded to a secure server via the transmitter up to 3 times per day when the vest was within range of the transmission device, circumventing the need for subjects or parents to manually transmit data. These data were collected for a 12-month period for each subject.

In addition to the vest usage data, we gathered subjectspecific data from the electronic medical record including demographics (ie, sex, age), vest therapy prescription data at the time of enrollment, changes in vest therapy prescription, and hospitalizations during the study period. For each subject, we excluded the first $7 \mathrm{~d}$ of vest-use data from analysis because these data would not accurately reflect vest use due to device setup as well as tests completed prior 
Vest Therapy Adherence Among Children With CF

Table 1. Demographics and Clinical Course of Subjects by Age Group

\begin{tabular}{|c|c|c|c|c|c|}
\hline & \multirow{2}{*}{ Overall } & \multicolumn{3}{|c|}{ Age Groups } & \multirow{2}{*}{$P$} \\
\hline & & $2-6 y$ & $7-12$ y & $13-19 y$ & \\
\hline Age & $9.6 \pm 5.2$ & $2.9 \pm 1.3$ & $9.1 \pm 1.9$ & $14.9 \pm 1.6$ & NA \\
\hline \multicolumn{5}{|l|}{ Gender } & .23 \\
\hline Female & $31(50.0)$ & $11(61.1)$ & $11(57.9)$ & $9(36.0)$ & \\
\hline Male & $31(50.0)$ & 7 (38.9) & $8(42.1)$ & $16(64.0)$ & \\
\hline Hospitalized ( $\geq 1$ time) & $16(25.8)$ & $2(11.1)$ & $6(31.6)$ & $8(32.0)$ & .23 \\
\hline Change in vest prescription & $18(29.0)$ & $6(33.3)$ & $7(36.8)$ & $5(20.0)$ & .46 \\
\hline
\end{tabular}

to shipment. Subject-level adherence calculations excluded periods when a subject was hospitalized. This was done because the study objective was to measure subject adherence at home. Adherence during a hospitalization would very likely be higher than when a subject was at home. Given that some subjects had a vest prescription change during the study, we measured adherence relative to the prescription in place at any given time.

\section{Outcomes}

Conceptually, we tend to think of adherence as binary: a patient is adherent to their prescribed treatment, or a patient is not. However, even if adherence is binary on a daily basis, adherence over a time is a continuous variable. Furthermore, a vest therapy prescription has several components that need to be considered for adherence. Prescription components include daily use, treatments per day, duration of treatment, and vest system settings (ie, frequency and pressure settings). We separately assessed adherence for each prescription component. For vest settings, we examined adherence to frequency settings alone and secondarily as a combination of frequency and pressure settings. For each prescription component, we computed mean adherence for the entire study period as well as on a weekly basis to assess time trends. For each component, the distribution was bimodal: one group of subjects with high adherence and another with low adherence. Accordingly, for each prescription component we classified subjects as adherent if they had a mean adherence $\geq 80 \%$ and as nonadherent if the mean was $<80 \%$. For example, a subject was classified as adherent to daily vest utilization if they used their vest on at least $80 \%$ of days during study.

In addition to assessing adherence for each prescription component, we created combined adherence metrics jointly considering adherence of $\geq 2$ individual prescription components to assess adherence more broadly. To achieve this, we ordered the facets of the prescription by presumed therapeutic importance. We considered adherence to daily use therapeutically the most important. Similarly, we considered adherence to the number of prescribed treatments per day of the next most importance. Hence, the first combined adherence metric included whether the subject was adherent to both daily use and the prescribed treatments per day. Next, we added adherence to prescribed treatment duration with daily treatment and treatments per day to assess the combined adherence to these 3 metrics. Finally, we added adherence to prescribed vest setting to assess overall adherence to all prescription components. This layering approach provided insight not only into adherence for specific prescription components but also into the level of adherence subjects achieved when the various components of the vest prescription were considered simultaneously.

\section{Data Analysis}

Demographic and clinical characteristics between age groups were compared using the Fisher exact test due to small cell counts. Each component of adherence was characterized using percent adherent. We summarized adherence for each prescription component individually as well as in conjunction with the other components. For each component, we used linear regression to assess time trends in weekly adherence rates. All analyses were performed using Stata 14 (StataCorp, College Station, Texas).

\section{Results}

Of 111 patients reviewed for eligibility, 91 remained after exclusion criteria were applied; of those, 9 declined to participate and 9 did not respond to outreach efforts by study staff. A total of 73 subjects were enrolled in the study; of those, 62 subjects (mean age $9.6 \mathrm{y}$, range 2-19 y) completed the study (Table 1). Those who did not complete the study were lost to follow-up prior to having the vest system set up in their homes. Study subjects overall were evenly split by gender, but there were some differences by age 


\section{Vest Therapy Adherence Among Children With CF}

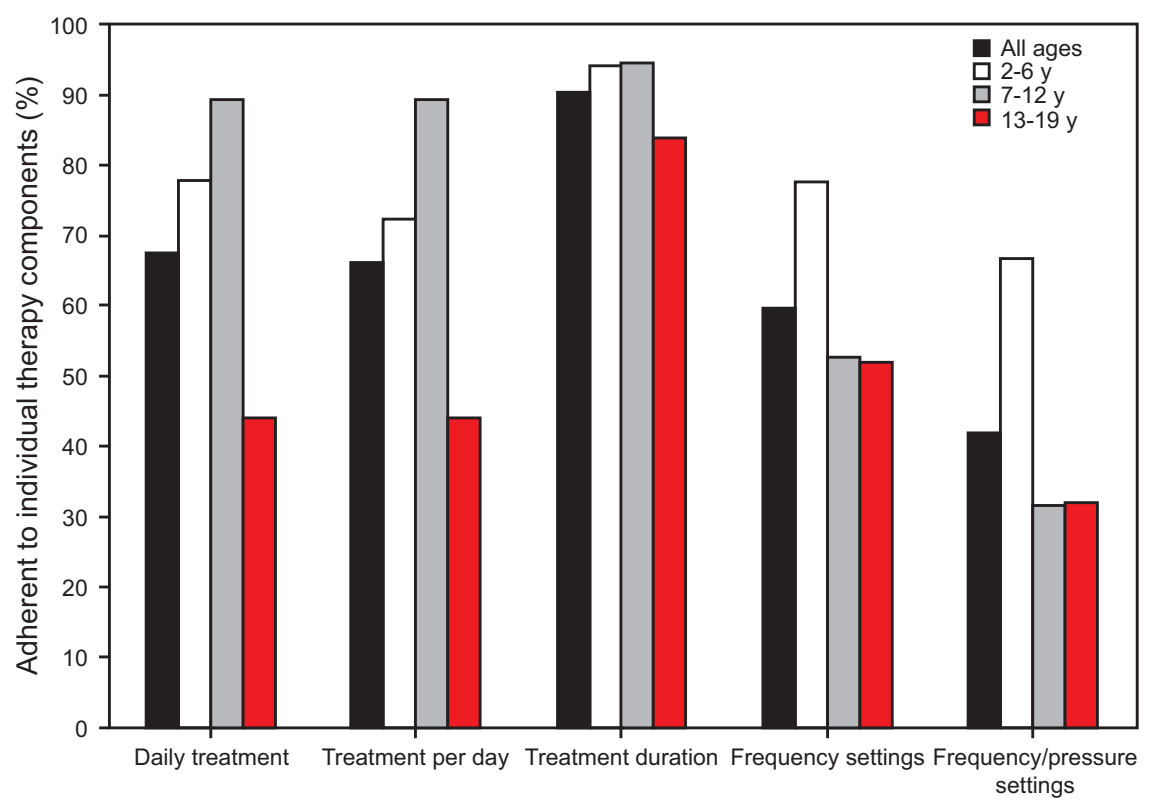

Fig. 1. Percent adherent ( $\geq 80 \%$ ) to individual therapy components by age group.

Table 2. Summary of Change in Mean Adherence Over Study Period*

\begin{tabular}{|c|c|c|c|c|}
\hline & Mean Adherence at Week 1 & Trend Coefficient & Cumulative Change & $P$ \\
\hline Daily treatment & $81.5 \%$ & -0.19 & $-9.7 \%$ & $<.001$ \\
\hline Treatments per day & $87.8 \%$ & 0.02 & $1.0 \%$ & .57 \\
\hline Treatment duration & $93.3 \%$ & -0.001 & $-0.1 \%$ & .94 \\
\hline Frequency settings & $76.7 \%$ & -0.04 & $-2.0 \%$ & .29 \\
\hline Frequency and pressure settings & $65.0 \%$ & -0.07 & $-3.6 \%$ & .16 \\
\hline
\end{tabular}

group. During the study period, $25 \%$ of subjects were hospitalized, and 29\% had a change in their vest prescription.

Figure 1 shows the percentage of subjects who were adherent to the various prescription components. For each component, adherence was lowest for those 13-19 y old. Subjects in this age group were significantly less adherent to daily treatment $(P=.004)$ and treatments per day $(P=$ .006) than subjects who were $7-12$ y old. Adherence to both daily treatment and the number of treatments per day was highest (89.5\%) for subjects 7-12 y old. Adherence to treatment duration was $90.3 \%$ across all subjects and did not differ significantly across age groups $(P=.49)$. For comparisons by age group (see Table 2 in the supplementary materials at http://www.rcjournal.com).

Figure 2 shows the combined adherence rates for $\geq 2$ prescription components. The adherence rates for daily treatment and number of treatments per day were both $89.5 \%$ for the 7-12 y age group (Fig. 1). Combining these 2 components revealed that $84.2 \%$ of these subjects were adherent to both. Adding additional components into the combined adherence metric resulted in a $21.1 \%$ adherence rate when all components were included for the 7-12 y age group. For subjects $2-6$ y old, $77.8 \%$ were adherent to daily treatment, but adherence dropped to $44.4 \%$ when all components were included in the adherence metric. Similarly, among those 13-19 y old, adherence to daily treatment was $44.0 \%$, but when all components were included in the combined metric, adherence was $4 \%$. Adherence rates between those 2-6 y old and those $7-12$ y old were not statistically different for the various combined adherence metrics except when all components were included in the combined metric (Table 2).

Results presented above show adherence over the entire study period. Figure 3 shows adherence trends in the various individual components over time. There was no significant time trend for treatments per day, treatment duration, or vest settings, but there was a significant $9.7 \%$ decrease $(P<.001)$ in adherence to daily treatment over the study (Table 2).

\section{Discussion}

With the availability of increasingly effective treatment options for cystic fibrosis, providers are faced with the 


\section{Vest Therapy Adherence Among Children With CF}

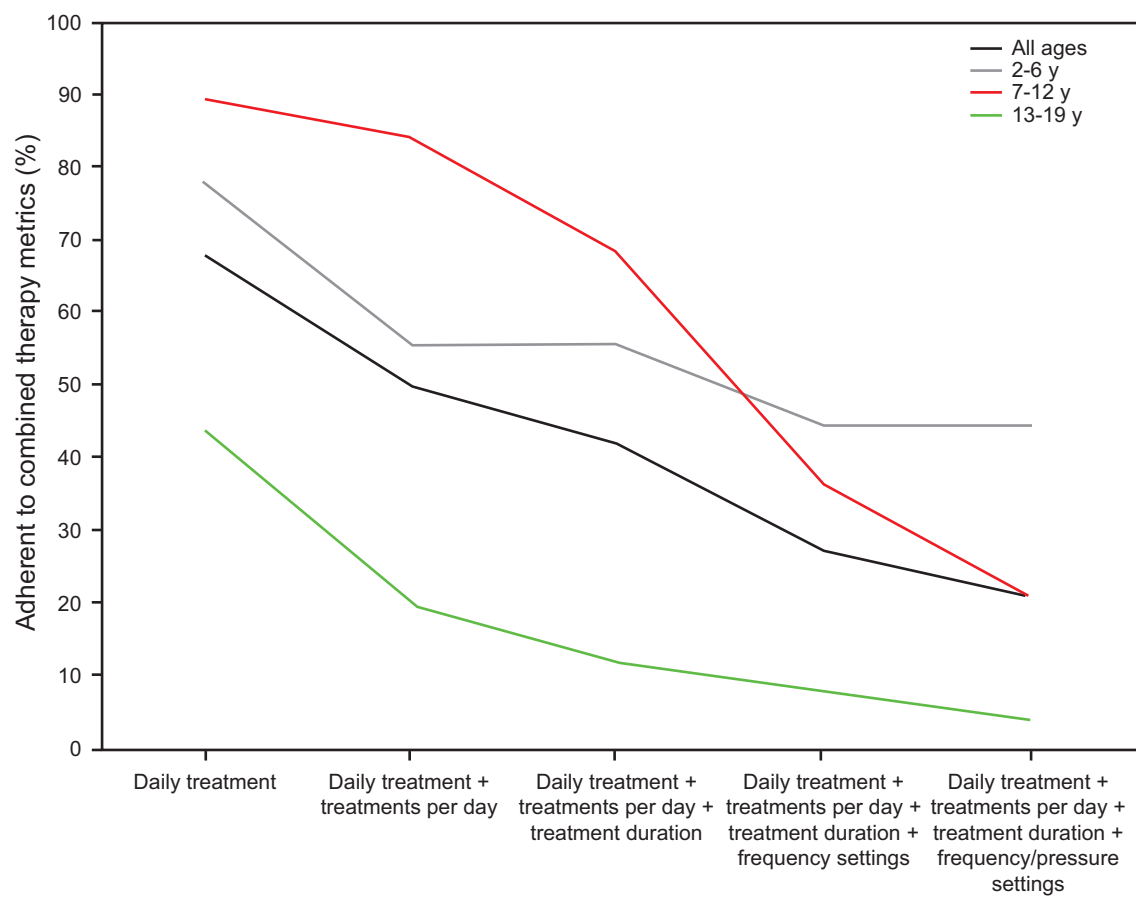

Fig. 2. Percent adherent ( $\geq 80 \%)$ to combined therapy component metrics by age group.

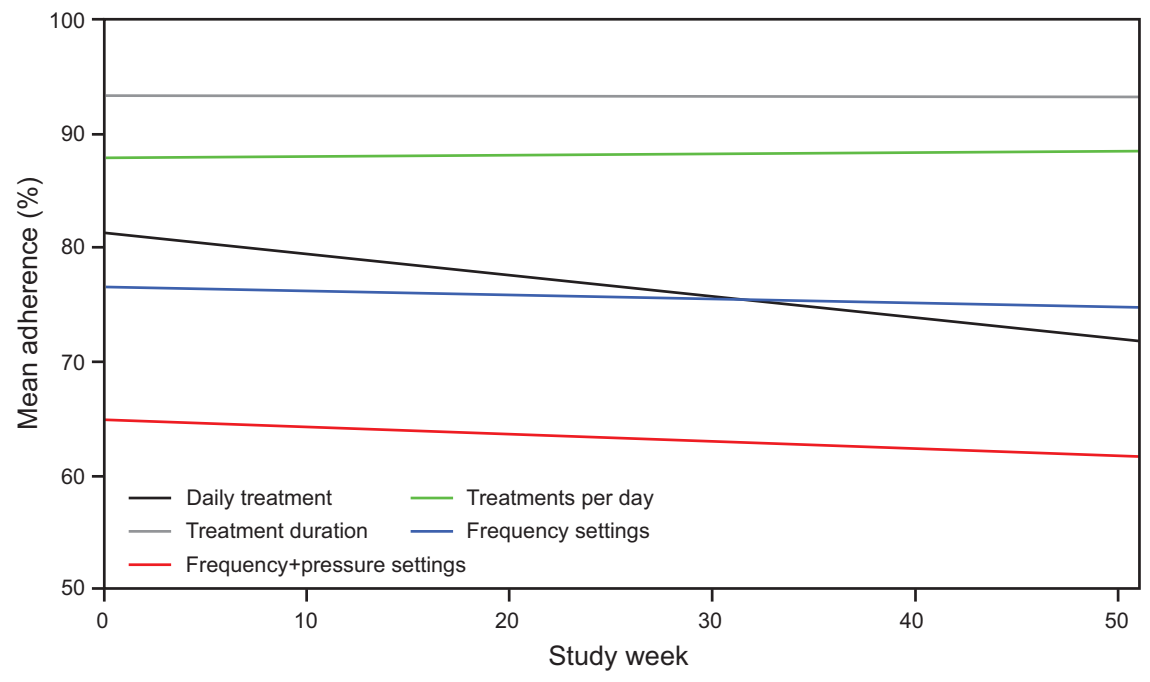

Fig. 3. Mean adherence to individual therapy components over the 51-week study period.

challenge of prescribing the most meaningful comprehensive treatment regimen while remaining sensitive to the burden that additional treatments place on patients and their families. Determining the impact of this time-consuming therapy on clinical outcomes may be confounded by how adherent a patient is to the prescribed treatment. Historically, providers have relied on patients' self-reported adherence and their own clinical judgment when assessing the effectiveness of vest therapy. However, past research indicates there are considerable discrepancies in self-reported adherence rates compared to rates measured with objective tools. ${ }^{1}$
With objective adherence data, providers can more effectively judge whether aspects of the vest prescription need to change to achieve desired clinical results or whether the clinical focus needs to be on better adherence. Further, with objective adherence data, future research can address the clinical effectiveness of individual aspects of the vest prescription that would not be possible with subjective, selfreported adherence. The Bluetooth-enabled system used in this study demonstrated a feasible way to remotely collect vest adherence data to aid providers in clinical judgments about the effectiveness of vest prescriptions. 


\section{Vest Therapy Adherence Among Children With CF}

Adherence rates for the adolescent age group (13-19 y of age) in this study were substantially below the other 2 age groups (2-6 y of age and 7-12 y of age), consistent with past studies of disease management and adherence among children and adolescents with chronic conditions such as cystic fibrosis and type 1 diabetes. ${ }^{8,9,15-18}$ Although adolescence is commonly a time for increased autonomy and self-determination, there is still a role for caregivers to play in support of therapeutic management for chronically ill adolescents. ${ }^{19-25}$ Our data suggest there may be a need for more frequent and open discussions between providers and caregivers regarding daily therapeutic routines, informed by objective usage data. Given the fact that adolescents who did the vest therapy generally were adherent to doing the treatment for the prescribed duration but were less adherent with the prescribed vest settings, care teams may be inclined to focus on the more detailed usage data when designing interventions. However, the greater challenge for this age group is that they were significantly less likely to use their vest and, as such, would likely benefit from a simpler regimen and consistent checkins by both their care team and their caregivers.

Our findings on daily adherence and combined adherence metrics by age group highlight areas that care teams can target to improve patient adherence to vest prescriptions. For children 7-12 y old, the group in which we found adherence to vest settings lowered overall adherence, it may be beneficial for care teams to request that patients bring the vest systems to annual clinical visits to ensure settings are appropriately programmed and that caregivers understand how to reprogram the software when the child's prescription changes. While this study notes that adherence was lacking in these areas, it does not answer the question of how successful efforts targeting these areas would be in improving adherence rates or clinical outcomes.

The time trend analysis, in which adherence to daily treatment fell while adherence to all other vest prescription aspects did not significantly change, may reflect surveillance bias in our study. Subjects who were aware their data were being collected remotely may have been more apt to use their vest early in the study. It may also indicate that daily use drops off over time, regardless of study participation. While conversations between care teams, patients, and caregivers regarding daily vest usage are already common, the downward time trend may suggest a need for more than clinical dialogue to maintain optimal adherence to daily treatment over time. Development of tools or games to promote therapy adherence is underway in other therapeutic areas and should be considered in support of vest therapy, as well. ${ }^{26-28}$

This study attempts to organize prescription components by therapeutic importance with daily treatment as the presumed most important factor. The trend analysis supports the assumption that regular intervention regarding daily use is the most important because daily treatment decreased over time while adherence was unchanged for other components. This suggests that simply doing the treatment is the greatest hurdle, given that once a patient starts a treatment, their pattern of adherence is consistent. Hence, adherence to prescription aspects such as duration and vest settings may remain high if patients and families become well-trained early on in their vest utilization.

Our study had several limitations. Subjects involved in this study were from a single clinical center and included only those who consented to be monitored and successfully completed the vest system set-up process. As such, results may not be generalizable to the pediatric cystic fibrosis community at large and may be inflated due to self-selection. Additionally, we collected data remotely, which allows for the chance that vest systems may have been run when the subjects were not wearing the vest and truly completing their therapy. Finally, there were limitations created by the role of third-party technicians in setting up vest systems. The research team did not control the initial vest system programming in order to remain consistent with the standard of care practice of having device technicians complete this step with families. Vest setting adherence may have been affected by how systems were originally programmed and how caregivers and subjects were trained.

\section{Conclusions}

This study has 3 principal contributions. First, it provides objective measures of vest prescription adherence using an automated, remote data collection mechanism. These data can be processed to provide objective, real-time information for providers to support clinical judgments about the efficacy of vest therapy in relation to patient adherence with prescribed treatment. Care teams can also use these data to target specific adherence gaps. Second, this study developed a framework to simultaneously assess adherence to multiple components of a vest prescription from daily treatment to vest settings, as well as a framework to combine these aspects into an overall adherence measure. Finally, the results showed significant differences in adherence patterns by age group. Care teams can use these age-specific trends to inform and tailor their approaches to working with both patients and caregivers to optimize their therapeutic practices throughout childhood and adolescence.

\section{ACKNOWLEDGMENTS}

We thank Brittany Machus and Sara Lee for their support in the study implementation, and Kristen Griffin for her thoughtful review of this manuscript and the revisions that she provided.

\section{REFERENCES}

1. O’Donohoe R, Fullen BM. Adherence of subjects with cystic fibrosis to their home program: a systematic review. Respir Care 2014;59 (11):1731-1746. 


\section{Vest Therapy Adherence Among Children With CF}

2. Riekert KA, Eakin MN, Bilderback A, Ridge AK, Marshall BC. Opportunities for cystic fibrosis care teams to support treatment adherence. J Cyst Fibros 2015; 14(1):142-148.

3. Beers MH, Berkow R. The Merck manual of diagnosis and therapy. Whitehouse Station, NJ: Merck \& Co. Inc; 1999.

4. MacKenzie T, Gifford AH, Sabadosa KA, Quinton HB, Knapp EA, Goss $\mathrm{CH}$, Marshall BC. Longevity of patients with cystic fibrosis in 2000 to 2010 and beyond: survival analysis of the Cystic Fibrosis Foundation Patient Registry. Ann Intern Med 2014;161(4):233-241.

5. Flume PA, Robinson KA, O'Sullivan BP, Finder JD, Vender RL, Willey-Courand D-B, et al. Cystic fibrosis pulmonary guidelines: airway clearance therapies. Respir Care 2009;54(4):522-537.

6. Modi AC, Lim CS, Yu N, Geller D, Wagner MH, Quittner AL. A multi-method assessment of treatment adherence for children with cystic fibrosis. J Cyst Fibros 2006;5(3):177-185.

7. Passero MA, Remor B, Salomon J. Patient reported compliance with cystic fibrosis therapy. Clin Pediatr (Phila) 1981;20(4):264-268.

8. White T, Miller J, Smith GL, McMahon WM. Adherence and psychopathology in children and adolescents with cystic fibrosis. Eur Child Adolesc Psychiatry 2009;18(2):96-104.

9. Quittner AL, Drotar D, Ievers-Landis CE, Seidner D, Slocum N, Jacobsen J. Adherence to medical treatments in adolescents with cystic fibrosis: the development and evaluation of family-based interventions. In: Drotar D, editor. Promoting adherence to medical treatment in childhood chronic illness: interventions and methods. Hillsdale, NJ: Erlbaum Associates; 2000:383-407.

10. Abbott J, Dodd M, Webb AK. Health perceptions and treatment adherence in adults with cystic fibrosis. Thorax 1996;51(12):1233-1238.

11. Conway SP, Pond MN, Hamnett T, Watson A. Compliance with treatment in adult patients with cystic fibrosis. Thorax 1996;51(1):29-33.

12. White D, Stiller K, Haensel N. Adherence of adult cystic fibrosis patients with airway clearance and exercise regimens. J Cyst Fibros 2007;6(3):163-170.

13. Volsko TA. Airway clearance therapy: finding the evidence. Respir Care 2013;58(10):1669-1678.

14. Mikesell CL, Kempainen RR, Laguna TA, Menk JS, Wey AR, Gaillard PR, et al. Objective measurement of adherence to out-patient airway clearance therapy by high-frequency chest wall compression in cystic fibrosis. Respir Care 2017;62(7):920-927.

15. Arias Llorente RP, Bousoño García C, Díaz Martín JJ. Treatment compliance in children and adults with cystic fibrosis. J Cyst Fibros 2008;7 (5):359-367.

16. Clements MA, Foster NS, Maahs DM, Schatz DA, Olson BA, Tsalikian E, et al. Hemoglobin A1C (HbA1c) changes over time among adolescent and young adult participants in the T1D exchange clinic registry. Pediatr Diabetes 2016;17(5):327-336.

17. Hilliard ME, Wu YP, Rausch J, Dolan LM, Hood KK. Predictors of deteriorations in diabetes management and control in adolescents with type 1 diabetes. J Adolesc Health 2013;52(1):28-34.

18. Zindani GN, Streetman DD, Streetman DS, Nasr SZ. Adherence to treatment in children and adolescent patients with cystic fibrosis. J Adolesc Health 2006;38(1):13-17.

19. Butcher JL, Nasr SZ. Direct observation of respiratory treatments in cystic fibrosis: parent-child interactions relate to medical regimen adherence. J Pediatr Psychol 2015;40(1):8-17.

20. Bucks RS, Hawkins K, Skinner TC, Horn S, Seddon P, Horne R. Adherence to treatment in adolescents with cystic fibrosis: the role of illness perceptions and treatment beliefs. J Pediatr Psychol 2009;34 (8):893-902.

21. Dziuban EJ, Saab-Abazeed L, Chaudhry SR, Streetman DS, Nasr SZ. Identifying barriers to treatment adherence and related attitudinal patterns in adolescents with cystic fibrosis. Pediatr Pulmonol 2010;45 (5):450-458

22. King PS, Berg CA, Butner J, Drew LM, Foster C, Donaldson D, et al. Longitudinal trajectories of metabolic control across adolescence: associations with parental involvement, adolescents' psychosocial maturity, and health care utilization. J Adolesc Health 2012;50(5):491496.

23. Rausch JR, Hood KK, Delamater A, Shroff Pendley J, Rohan JM, Reeves G, et al. Changes in treatment adherence and glycemic control during the transition to adolescence in type 1 diabetes. Diabetes Care 2012;35(6):1219-1224.

24. Helgeson VS, Reynolds KA, Siminerio L, Escobar O, Becker D. Parent and adolescent distribution of responsibility for diabetes selfcare: Links to health outcomes. J Pediatr Psychol 2008;33(5):497-508.

25. King PS, Berg CA, Butner J, Butler JM, Wiebe DJ. Longitudinal trajectories of parental involvement in type 1 diabetes and adolescents' adherence. Health Psychol 2014;33(5):424-432.

26. Balli F. Developing digital games to address airway clearance therapy in children with cystic fibrosis: participatory design process. JMIR Serious Games 2018;6(4):e18.

27. Whiteley L, Brown LK, Mena L, Craker L, Arnold T. Enhancing health among youth living with HIV using an iPhone game. AIDS Care 2018;30(Suppl 4):21-33.

28. Joubert M, Armand C, Morera J, Tokayeva L, Guillaume A, Reznik Y. Impact of a serious videogame designed for flexible insulin therapy on the knowledge and behaviors of children with type 1 diabetes: the LUDIDIAB pilot study. Diabetes Technol Ther 2016;18(2):52-58. 